\title{
DBI analog of a decaying vacuum cosmology
}

\author{
Dennis Bessada ${ }^{1,2},{ }^{*}$ \\ ${ }^{1}$ Laboratório de Física Teórica e Computação Científica, \\ Universidade Federal de São Paulo - UNIFESP, Campus Diadema, Brazil \\ ${ }^{2}$ INPE - Instituto Nacional de Pesquisas Espaciais - Divisão de Astrofísica, São José dos Campos, 12227-010 SP, Brazil
}

\begin{abstract}
In this work I discuss the dynamical and thermodynamical equivalence between a general $k$ essence scalar field cosmology and an arbitrary cosmological model with a decaying vacuum, thus generalizing the approach proposed by Maia and Lima [Phys. Rev. D 65, 083513 (2002)]. The formalism obtained is quite general and holds for any non-canonical scalar field model. As a special case I derive a Dirac-Born-Infeld (DBI) model with an exponential potential and constant speed of sound, and show that it is equivalent to a cosmological model with decay law $\Lambda(H)=3 \beta H^{2}$.
\end{abstract}

PACS numbers: 98.80.Hw, 04.40.Nr, 95.35.+d

\section{INTRODUCTION}

The discovery of cosmic acceleration [1] opened up a new research field in cosmology. Many models have been proposed to account for this observational evidence, and the dark energy (DE) hypothesis, in which a fluid with negative pressure is responsible for driving cosmic acceleration, seems to be the most promising candidate. However, the physical nature of DE is still obscure. The most accepted paradigm establishes the cosmological constant $\Lambda$ (CC for short) as playing the role of such "fluid" with negative pressure, dominating the energy density of the universe today. Despite fitting well the available data, this approach is plagued with some problems (see [2] for a discussion); among them, it is worth recalling the the so-called fine-tuning problem, (or the old CC problem) and the coincidence problem. The first one is related to the fact that the present-time observed value for the vacuum energy density, $\rho_{\Lambda}=\Lambda c^{2} /(8 \pi G) \sim 10^{-47} \mathrm{GeV}^{4}$, is more than 100 orders of magnitude smaller the value found using the methods of quantum field theory (QFT) $\left(\sim 10^{71} \mathrm{GeV}^{4}\right)[3]$. The coincidence problem is related to the fact that the vacuum energy density started to dominate over the matter energy density just at the present cosmological time.

A natural attempt to alleviate both problems relies on the introduction of additional dynamics to the cosmological model in order to make the vacuum energy density evolve with time; thus, the corresponding vacuum energy density would have a high enough value to drive inflation at the very early universe, decaying along the expansion history to its small value observed today. Such mechanism can be implemented with the help of scalar fields [4], for example; in this case, there is a phase in which the potential dominates over the kinetic energy, leading to the desired negative pressure to drive cosmic acceleration. Also, along with this "canonical" scenario there is also the so-called "noncanonical" approach, in which the

\footnotetext{
*dennis. bessada@unifesp.br
}

scalar field Lagrangian exhibits a nonlinear kinetic term. This class of models, also called $k$-essence, appeared first in the context of inflation [5]. It was later generalized to account for the cosmic acceleration [6], and thereafter many proposals have been done in the literature following this path. Some of them are derived as low-energy solutions in string theories, and the possibility of being derived from a more fundamental theory is one of their attractive features, provided they solve the same puzzles as the conventional theories do. One of the most promising string-inspired proposals is the so-called DiracBorn-Infeld (DBI) model [7, 8], which comes out from a D3-brane motion within a warped compactification. DBI models can provide very interesting inflationary (see, for example, 9 and references therein), as well as DE-like solutions [10 12]. In particular, in DBI inflation the inflaton field is interpreted as the distance between two branes moving in the extra dimensions along a warped throat; thus, instead of being inserted phenomenologically in the Lagrangian, the inflaton actually emerges from fundamental physics in this scenario.

As an alternative to the scalar field approach, it is also possible to introduce additional dynamics to the cosmological model by means of a phenomenological timedependent cosmological term $\Lambda(t)$ 13 18] (see also 19] for a discussion of $\Lambda(t)$ models arising in the context of QFT in curved space-time). In this scenario, the timedependent cosmological term yields a coupling with another cosmic component, which implies either particle production or an increase in the time-varying mass of the dark matter particles 20$]$.

Since $\Lambda(t)$ models are essentially of phenomenological nature, it is of extreme importance to derive them from fundamental physics to allow, for example, their embedding in high-energy theories or in modified gravity models as suggested in DBI models. A first step towards this goal has been achieved in [21], where the authors derived a classical scalar field model with dynamical and thermodynamical properties equivalent to those of some particular $\Lambda(t)$ models. Among other results, they have found an equivalence between a decaying vacuum model ruled by the law $\Lambda(H)=3 \beta H^{2}$ and a scalar field model with an exponential potential. The key to this 
connection lies on two fundamental assumptions: first, that both approaches obey the same dynamical equations parametrized by a factor $\bar{\gamma}$, which depends on the specific $\Lambda(t)$ model adopted; second, they follow the same equilibrium thermodynamical equations (deduced in 22]) for the source terms (which represent the particle creation process) and for the temperature laws. A natural generalization of such methods would include noncanonical scalar fields, and this is precisely the main goal of this work. The equations derived here are quite general, and hold for any noncanonical field; also, I show that all the results obtained in this paper reduce to those found in [21]. As a noncanonical example I choose a DBI model with a constant speed of sound and exponential potential, and show that it is dynamical- and thermodynamically equivalent to a decaying-vacuum model with the law $\Lambda(H)=3 \beta H^{2}$.

The present paper is organized as follows: in Section IIA I review the basics of cosmological models with vacuum decay, whereas in Section IIBI discuss their thermodynamical properties. In Section III I review the basics of noncanonical scalar field models and set up the dynamical and thermodynamical equivalence between such class of models and the decaying vacuum ones. In Section IVB I derive a particular DBI model to illustrate the methods developed.

\section{COSMOLOGICAL MODELS WITH A DECAYING VACUUM}

\section{A. The background equations}

Throughout this paper I consider a flat, homogeneous and isotropic universe described by the FriedmanRobertson-Walker (FRW) metric

$$
d s^{2}=d t^{2}-a(t)^{2}\left(d r^{2}+r^{2} d \theta^{2}+r^{2} \sin ^{2} \theta d \phi^{2}\right),
$$

and filled with a perfect fluid with energy density $\rho$ and pressure $P$, whose stress energy-momentum tensor is given by

$$
T_{m}^{\alpha \beta}=(\rho+P) u^{\alpha} u^{\beta}-P g^{\alpha \beta},
$$

where $u^{\alpha}$ is the fluid four-velocity. In this picture, it is assumed that the perfect fluid pressure and energy density are connected via the equation of state

$$
P=w \rho=(\gamma-1) \rho,
$$

where $\gamma$ is the barotropic index.

In the presence of a cosmological term $\Lambda$, Einstein field equations read

$$
G^{\alpha \beta}-\Lambda g^{\alpha \beta}=\kappa^{2} T_{m}^{\alpha \beta},
$$

where $\kappa^{2} \equiv M_{P}^{-2} \equiv 8 \pi G, M_{P}$ being the reduced Planck mass. Taking the covariant divergence of both sides of equation (4), and assuming that the fluid energymomentum satisfies the conservation law $T_{m ; \beta}^{\alpha \beta}=0$, it follows that the covariant divergent of $\Lambda$ also vanishes, since, by the Bianchi identities, $G_{; \beta}^{\alpha \beta}=0$, which sets $\Lambda$ to be a constant. In this picture, the cosmological constant $\Lambda$ is a purely geometrical entity; however, if we move the cosmological term to the right-hand side of the same equation, and interpreting $\Lambda$ as a second fluid, there is no further reason to keep this term constant with respect to time. In this case, we define the effective energymomentum tensor for the two fluids as

$$
\bar{T}^{\alpha \beta} \equiv T_{m}^{\alpha \beta}+M_{P}^{2} \Lambda g^{\alpha \beta},
$$

which naturally satisfies the energy and momentum conservation constraint

$$
\bar{T}_{; \beta}^{\alpha \beta}=0
$$

as a consequence of the Bianchi identities.

Next, substituting the metric (11) into (4), we get the Friedman equations

$$
\begin{aligned}
\kappa^{2} \rho+\Lambda & =3 H^{2}, \\
\kappa^{2} P-\Lambda & =-2 \dot{H}-3 H^{2},
\end{aligned}
$$

where $H=\dot{a} / a$ is the Hubble parameter; also, from the energy conservation constraint (6) we get the continuity equation

$$
\dot{\rho}+3 H(\rho+P)=-M_{P}^{2} \dot{\Lambda} .
$$

Hence, a cosmological model with varying $\Lambda$ implies that energy is transferred from this cosmological term to the perfect fluid; in other words, the vacuum content decays into particles. Although particle creation usually leads to nonequilibrium processes, it is also possible to find a particular configuration of the system in which equilibrium relations still hold, as we shall discuss in Section II .

Next, adding up equations (7) and (8), we get the expression

$$
\kappa^{2}(\rho+P)=-2 \dot{H}
$$

which yields, using equations (3) and (7),

$$
\frac{2}{3} \frac{\dot{H}}{H^{2}}=-\bar{\gamma},
$$

where we have defined [21]

$$
\bar{\gamma} \equiv \gamma\left(1-\frac{\Lambda}{3 H^{2}}\right) .
$$

The function $\bar{\gamma}$ correlates the time dependence of each $\Lambda(t)$ model with the Hubble parameter and its derivative. This fact is very convenient to our purposes, for given a particular $\Lambda(t)$ model, we can derive the corresponding expression for $\bar{\gamma}$ by means of equation (12), 
and use it to solve equation (11) for its scalar-field analog. We shall address this correspondence in Sections III and IV A, IVB.

In terms of this quantity, Friedman equation (77) yields the following expression for the fluid energy density

$$
\rho=3 M_{P}^{2} H^{2} \frac{\bar{\gamma}}{\gamma}
$$

next, defining the source term for the cosmological "fluid" $\Lambda$ appearing in equation (9) as

$$
F \equiv-M_{P}^{2} \dot{\Lambda}
$$

we see that it is related to $\bar{\gamma}$ through

$$
F=3 H \gamma \rho\left(1-\frac{\bar{\gamma}}{\gamma}+\frac{1}{3 H \gamma} \frac{\dot{\bar{\gamma}}}{\bar{\gamma}}\right)
$$

where we have used (9), (11) and (13).

\section{B. Thermodynamical properties of $\Lambda$-decaying models}

As I have briefly mentioned in the last section, particle creation usually leads to non-equilibrium processes. However, under some simple conditions, we can derive a temperature-evolution law for vacuum-decaying models with solely the equilibrium contribution. In this section I deal with this issue, following closely [22].

The macroscopic variables that completely describe the thermodynamical states of a relativistic perfect fluid are given by the energy-momentum tensor $T_{m}^{\alpha \beta}$, the entropy current $S^{\alpha}$ and the particle current $N^{\alpha}$. Introducing the particle number density $n$ and the specific entropy per particle $\sigma$, the entropy and particle number density currents are given respectively by

$$
S^{\alpha} \equiv n \sigma u^{\alpha}
$$

and

$$
N^{\alpha} \equiv n u^{\alpha}
$$

The covariant divergence $N^{\alpha}$; leads to the balance equation

$$
\frac{\dot{N}}{N}=\frac{\dot{n}}{n}+3 H \equiv \Gamma,
$$

where $\Gamma$ is the particle creation rate in a comoving volume. As for the entropy current, the second law of thermodynamics implies that $S^{\alpha} ; \alpha \geq 0$.

Next, using Gibbs's relation

$$
n T d \sigma=d \rho-\frac{\rho+P}{n} d n
$$

where $T$ is the temperature, and using equations (9), (14) and (18) we have that

$$
\dot{\sigma}=\frac{1}{n T}[F-(\rho+P) \Gamma]
$$

then, from this equation plus $S^{\alpha}{ }_{; \alpha}=\dot{n} \sigma+n \dot{\sigma}+3 H n \sigma$, it can be shown that the temperature evolution law for the relativistic fluid reads

$$
\frac{\dot{T}}{T}=\left(\frac{\partial P}{\partial \rho}\right)_{n} \frac{\dot{n}}{n}+\frac{\dot{\sigma}}{(\partial \rho / \partial T)_{n}} .
$$

Notice that, if the specific entropy is constant, one has $\dot{\sigma}=0$, so that

$$
F=\gamma \rho \Gamma
$$

for a fluid obeying the equation of state (3). Also, we get from (21) the usual equilibrium law

$$
\frac{\dot{T}}{T}=\left(\frac{\partial P}{\partial \rho}\right)_{n} \frac{\dot{n}}{n}
$$

whose integration yields the usual equilibrium relation for the particle number density

$$
n(T) \propto T^{1 /(\gamma-1)} .
$$

Also, in this "adiabatic" case, substituting equations (18) and (22) into the continuity equation (9), we get

$$
\rho(T) \propto T^{\gamma /(\gamma-1)} .
$$

Hence, even in a vacuum-decaying model the usual equilibrium thermodynamical relations hold if we assume that the specific entropy is constant. This is valid if we assume that the perfect fluid representing the matter or radiation content of the universe obeys the equation of state (3) and its number particle density and energy density are given by the equilibrium equations (24) and (25) respectively.

We are able now to relate the equilibrium temperature law (23) to the expression of the source term $F$ in terms of $\bar{\gamma}$, equation (15). We can do this by substituting equations (3), (18), and (22) in (23), which yields

$$
\frac{\dot{T}}{T}=-3 H \frac{\gamma-1}{\gamma}\left[\bar{\gamma}-\frac{1}{3 \gamma H} \frac{\dot{\bar{\gamma}}}{\bar{\gamma}}\right] \text {. }
$$

\section{NON-CANONICAL SCALAR FIELD MODELS}

In order to generalize the canonical scalar field description proposed in [21], let us introduce some important concepts concerning noncanonical models, or $k$-essence models [5]. Given a scalar field $\phi$ and the canonical $k i$ netic term $X \equiv \partial_{\alpha} \phi \partial^{\alpha} \phi / 2$, a general $k$-essence field theory is characterized by a noncanonical kinetic term $F(X)$ in its Lagrangian, where $F$ is an arbitrary function of $X$ (see [23] and references therein). The action of a $k$ essence field minimally coupled with gravity and a perfect fluid is then given by

$$
S=\int d^{4} x \sqrt{-g}\left[\frac{M_{P}^{2}}{2}+\mathcal{L}(X, \phi)+\mathcal{L}_{m}\right]
$$


from this action we can derive the expression of the energy-momentum tensor for the noncanonical field,

$$
T_{\phi}^{\alpha \beta}=\left(\rho_{\phi}+P_{\phi}\right) u^{\alpha} u^{\beta}-P_{\phi} g^{\alpha \beta},
$$

where the field energy density $\rho_{\phi}$ and pressure $P_{\phi}$ are given by

$$
\begin{aligned}
\rho_{\phi}(X, \phi) & =2 X \mathcal{L}_{X}-\mathcal{L}(X, \phi), \\
P_{\phi}(X, \phi) & =\mathcal{L}(X, \phi),
\end{aligned}
$$

and

$$
u_{\alpha}=\frac{\partial_{\alpha} \phi}{\sqrt{2 X}} .
$$

The subscript " $X$ " denotes a derivative with respect to the kinetic term $X$. For a homogeneous field $\phi$ and a FRW background, the energy-momentum tensor for the background and the $k$-essence fluids is given by

$$
\begin{aligned}
T_{\phi}^{\alpha \beta} & =\left(\rho_{B}+\rho_{\phi}+P_{B}+P_{\phi}\right) u^{\alpha} u^{\beta} \\
& -\left(P_{B}+P_{\phi}\right) g^{\alpha \beta}
\end{aligned}
$$

where the subscript " $B$ " stands for the background fluid. I use such label to distinguish the fluid component quantities in the noncanonical field description from their $\Lambda(t)$ counterparts. From this tensor we derive the Friedman equations for this two-fluid model, which read

$$
\begin{aligned}
\kappa^{2}\left(\rho_{B}+\rho_{\phi}\right) & =3 H^{2}, \\
\kappa^{2}\left(P_{B}+P_{\phi}\right) & =-2 \dot{H}-3 H^{2} ;
\end{aligned}
$$

the continuity equation comes from the constraint (6) applied to (32):

$$
\dot{\rho}_{B}+3 H\left(\rho_{B}+P_{B}\right)=-\dot{\rho}_{\phi}-3 H\left(\rho_{\phi}+P_{\phi}\right),
$$

which can be rewritten as

$$
\dot{\rho}_{B}+3 H\left(\rho_{B}+P_{B}\right)=\mathcal{F},
$$

where

$$
\mathcal{F} \equiv-\dot{\rho}_{\phi}-3 H\left(\rho_{\phi}+P_{\phi}\right) \text {. }
$$

Taking the derivative of (29) with respect to the cosmic time $t$, using the identity

$$
\frac{d}{d t}=\dot{X} \frac{\partial}{\partial X}+\sqrt{2 X} \frac{\partial}{\partial \phi}
$$

and the expression for the speed of sound for a $k$-essence field,

$$
c_{s}^{2}=\left(1+2 X \frac{\mathcal{L}_{X X}}{\mathcal{L}_{X}}\right)^{-1},
$$

we find that the source term for particle creation due to the decay of the noncanonical field is given by

$$
\begin{aligned}
\mathcal{F} & =-\frac{\dot{X} \mathcal{L}_{X}}{c_{s}^{2}}-\sqrt{2 X}\left(2 X \mathcal{L}_{X \phi}+3 H \sqrt{2 X} \mathcal{L}_{X}\right. \\
& \left.-\mathcal{L}_{\phi}\right) .
\end{aligned}
$$

As we have discussed in Section IA $\bar{\gamma}$ is an important parameter to specify the cosmological dynamics in terms of a given $\Lambda(t)$ model. In order to find its noncanonical scalar field analog, we must rewrite the dynamical equations of such field in terms of the parameter $\bar{\gamma}$; to do so, we first introduce the equation of state $P_{B}=(\gamma-1) \rho_{B}$ for the background fluid, and then add equations (33) 34 , so that

$$
\rho_{B}=3 M_{P}^{2} H^{2} \frac{\bar{\gamma}}{\gamma}-\frac{\left(\rho_{\phi}+P_{\phi}\right)}{\gamma},
$$

where we have used the definition for $\bar{\gamma}$, equation (12). Next, introducing the total energy density $\bar{\rho}$ as

$$
\bar{\rho} \equiv \rho_{\phi}+\rho_{B}=3 M_{P}^{2} H^{2}
$$

and defining the new variable $x$ as

$$
x \equiv \frac{\rho_{\phi}+P_{\phi}}{\bar{\rho} \bar{\gamma}},
$$

we see from (41) that

$$
\rho_{B}=\frac{\bar{\rho} \bar{\gamma}}{\gamma}(1-x)
$$

The quantity $x$ defined in (43) generalizes its canonical analog introduced in [21]. In order to understand its physical meaning, since it is going to play an important role in our discussion, let us rewrite this term in the following way: we add again equations (33) and (34) and use (12) and (42), so that

$$
\bar{\rho}+\bar{P}=-2 M_{P}^{2} \dot{H}=\bar{\rho} \bar{\gamma},
$$

where we have defined $\bar{P} \equiv P_{B}+P_{\phi}$. Hence, in this formulation the $x$ parameter assumes the form

$$
x=\frac{\rho_{\phi}+P_{\phi}}{\bar{\rho}+\bar{P}}
$$

thus, it measures the relative weight of the noncanonical scalar field energy density and pressure contribution with regard to the total energy density of the universe. Note that if $x=0$, one has, from equations (29130),

$$
\rho_{\phi}+P_{\phi}=2 X \mathcal{L}_{X}=0
$$

which leads to $X=0$. Hence, in this case, $P_{B}=-\rho_{B}$, recovering the $\Lambda(t)$ scenario. If $x=1$, we have $\rho_{B}=$ 0 , which leads to an universe whose evolution is driven solely by a $k$-essence field.

Notice that this result is similar to the one obtained in 21]; actually, by setting $\mathcal{L}(X, \phi)=X-V$ in (27) (which corresponds to the canonical limit), we get equation (22) in that reference, as expected.

We next turn to the thermodynamical analysis of the particle creation for the $k$-essence case. As pointed out by 
[21], the parameter $x$ plays an important role in the thermodynamics of vacuum decay; for instance, the source term (37) reads

$$
\mathcal{F}=3 H \gamma \rho_{B}\left[1-\frac{\bar{\gamma}}{\gamma}+\frac{1}{3 H \gamma}\left(\frac{\dot{\bar{\gamma}}}{\bar{\gamma}}-\frac{\dot{x}}{1-x}\right)\right],
$$

where we have used equations (36) and (44) and the equation of state for the background fluid. Note that this expression for the source term as a function of the $x$ parameter appears to be the same as obtained in equation (29) of 21]; however, such resemblance is solely functional, for the differences show up when we write down the specific expression for $x$ in terms of $\rho_{\phi}$ and $P_{\phi}$, which are model dependent.

Since we are assuming that the $k$-essence field decays into particles as seen from the continuity equation (35), it is important to place the usual thermodynamical constraints on such process. As in Section IIB, we introduce the corresponding entropy and number density currents for the noncanonical field decay into matter particles as

$$
\mathcal{S}^{\alpha} \equiv \nu \varsigma u^{\alpha}
$$

and

$$
\mathcal{N}^{\alpha} \equiv \nu u^{\alpha}
$$

where $\nu$ is the particle number density and $\varsigma$ is the specific entropy for this process. As in (18), the balance equation for the noncanonical case comes from the covariant divergence $\mathcal{N}^{\alpha} ; \alpha$,

$$
\frac{\dot{\mathcal{N}}}{\mathcal{N}}=\frac{\dot{\nu}}{\nu}+3 H \equiv \bar{\Gamma},
$$

where now $\bar{\Gamma}$ is the particle creation rate in a comoving volume due to the noncanonical scalar field decay. Next, substituting equations (19), (36) into and (51) into $\mathcal{S}^{\alpha}{ }_{; \alpha}$ one gets a similar equation for the specific entropy time evolution,

$$
\dot{\varsigma}=\frac{1}{\nu \mathcal{T}}\left[\mathcal{F}-\gamma \rho_{B} \bar{\Gamma}\right]
$$

where $\mathcal{T}$ is the temperature associated with the decay. Assuming that the laws (24) and (25) also hold for the background fluid, the specific entropy $\varsigma$ is also conserved in the noncanonical description. Hence, from (52),

$$
\mathcal{F}=\gamma \rho_{B} \bar{\Gamma}
$$

In this "adiabatic" case, the temperature law is derived in the same way as done in Section $1 \mathrm{~B}$, and is given by

$$
\frac{\dot{\mathcal{T}}}{\mathcal{T}}=(\gamma-1) \frac{\dot{\nu}}{\nu}
$$

which, in terms of the $\bar{\gamma}$ parameter, reads

$$
\frac{\dot{\mathcal{T}}}{\mathcal{T}}=-3 H \frac{\gamma-1}{\gamma}\left[\bar{\gamma}-\frac{1}{3 \gamma H}\left(\frac{\dot{\bar{\gamma}}}{\bar{\gamma}}-\frac{\dot{x}}{1-x}\right)\right] .
$$

Expressions (48) and (55) contain an extra $\dot{x}$ contribution absent in the $\Lambda(t)$ scenario as seen from (15) and (26). If we set $x=$ const., we see that the terms inside the brackets in equations (15) and (53) are the same, so that

$$
\frac{\mathcal{F}}{\rho_{B}}=\frac{F}{\rho}
$$

which implies

$$
\Gamma=\bar{\Gamma}
$$

The equality above demonstrates that the "adiabatic" condition provides the equivalence mechanism of particle production: the decay of the vacuum is the same process as the decay of the noncanonical field.

Next, comparing the temperature laws (26) and (55) for $x=$ const., we see that the terms inside brackets are also equal, thus

$$
\frac{\dot{\mathcal{T}}}{\mathcal{T}}=\frac{\dot{T}}{T} \Longrightarrow \mathcal{T}=b T
$$

where $b$ is a proportionality constant that can be determined as follows: from (13) and (44) we see that

$$
\rho_{B}=\rho(1-x),
$$

and from (25) we obtain

$$
\frac{\rho_{B}}{\rho}=\left(\frac{\mathcal{T}}{T}\right)^{\gamma /(\gamma-1)}=(1-x),
$$

so that $b=(1-x)^{(\gamma-1) / \gamma}$, which yields the following connection between the temperatures of both scenarios:

$$
\mathcal{T}=T(1-x)^{(\gamma-1) / \gamma} .
$$

The particle number densities $n$ and $\nu$ can be related by using (24) and (61):

$$
\nu=n(1-x)^{1 / \gamma}
$$

As for the entropies of both scenarios, the "adiabatic" condition implies, for $\sigma=S / N$ and $\varsigma=\mathcal{S} / \mathcal{N}$, that

$$
\frac{\dot{N}}{N}=\frac{\dot{\mathcal{S}}}{\mathcal{S}}=\frac{\dot{S}}{S}
$$

The assumption $x=$ const. brings about two important consequences to the approach developed in this paper: first, it demonstrates that the $\Lambda(t)$ and the $k$-essence scenarios are equivalent from the thermodynamical point of view. Also, they have equivalent dynamics, for the details of the vacuum decay, expressed by $\bar{\gamma}$, also takes part of the dynamics of the corresponding noncanonical field.

Second, the canonical and all other noncanonical models have the same source term and obey the same temperature law, provided they decay into the same background fluid. Hence, all the different noncanonical models are 
also thermodynamically equivalent. This holds because, regardless of the functional form of $\rho_{\phi}$ and $P_{\phi}$, which are model dependent, the sum $\rho_{\phi}+P_{\phi}$ will be always proportional to $\gamma \rho_{B}$ :

$$
\rho_{\phi}+P_{\phi}=\frac{x}{1-x} \gamma \rho_{B} .
$$

Notice that all the results obtained here for an arbitrary noncanonical scalar field generalize those found in reference [21], since a canonical scalar field is the simplest nontrivial particular case of noncanonical models.

\section{IMPLEMENTING A DBI MODEL WITH VACUUM DECAY}

\section{A. The general theory}

As an application of the above formalism I specialize to a DBI model. Originally, the DBI Lagrangian emerged from the context of warped D-brane inflation, in which the inflationary mechanism is regarded as the motion of a D3-brane in a six-dimensional "throat" characterized by the metric 24]

$$
d s_{10}^{2}=h^{2}(r) d s_{4}^{2}+h^{-2}(r)\left(d r^{2}+r^{2} d s_{X_{5}}^{2}\right),
$$

where $h$ is the warp factor, $X_{5}$ is a Sasaki-Einstein fivemanifold which forms the base of the cone, and $r$ is the radial coordinate along the throat. In this case, the inflaton field $\phi$ is identified with $r$ as $\phi=\sqrt{T_{3}} r$, where $T_{3}$ is the brane tension. The dynamics of the D3-brane in the warped background (65) is then dictated by the DBI Lagrangian

$$
\mathcal{L}=-f^{-1}(\phi) \sqrt{1-2 f(\phi) X}-f^{-1}(\phi)-V(\phi),
$$

where $f^{-1}(\phi)=T_{3} h(\phi)^{4}$ is the inverse brane tension and $V(\phi)$ is an arbitrary potential. From the DBI Lagrangian (66) the speed of sound is given by

$$
c_{s}(\phi)=\sqrt{1-2 f(\phi) X},
$$

where I have used (39), whereas the energy density and pressure for the DBI field are given by

$$
\begin{gathered}
\rho_{\phi}=\frac{1-c_{s}}{c_{s} f}+V, \\
P_{\phi}=\frac{1-c_{s}}{f}-V,
\end{gathered}
$$

respectively, derived from equations (29) and (30). Next, adding up expressions (68) and (69), and using (67), we find that

$$
\rho_{\phi}+P_{\phi}=\frac{2 X}{c_{s}} .
$$

The potential can also be read from equations (29) and (30):

$$
V(\phi)=\frac{c_{s} \rho_{\phi}-P_{\phi}}{1+c_{s}}
$$

Such equations hold for any DBI model; thus, in order to write them as an analog of a specific $\Lambda(t)$ scenario, we must rewrite such equations in terms of the $x$ parameter, defined by expression (43). First, using (42) and (44), we find that

$$
\rho_{\phi}=\bar{\rho}+\frac{\bar{\rho} \bar{\gamma}}{\gamma}(x-1)
$$

and from equations (43), (68) and (70) we get the expression for the pressure of the noncanonical field,

$$
P_{\phi}=\bar{\rho}\left[-1+\bar{\gamma} x+\frac{\bar{\gamma}}{\gamma}(1-x)\right] .
$$

Substituting (70) into (43), we obtain the following equation for the kinetic term $X$ :

$$
X=\frac{3}{2} M_{P}^{2} H^{2} \bar{\gamma} c_{s} x
$$

whereas the potential comes from equations (71), (72) and (73):

$$
V(\phi)=\bar{\rho}\left[1-\bar{\gamma}\left(\frac{x}{1+c_{s}}+\frac{1-x}{\gamma}\right)\right] .
$$

Using the definition for $X$, equation (74) can be integrated to find the $a$ dependence of the noncanonical field $\phi:$

$$
\phi-\phi_{*}=\sqrt{3} M_{P} \int_{a_{*}}^{a} \sqrt{\bar{\gamma} c_{s} x} \frac{d a}{a},
$$

where $\phi>\phi_{*}$. The integral on the right-hand side of equation (76) depends on the details of the underlying DBI model and on the explicit time dependence of $\Lambda$ due to the presence of $\bar{\gamma}$. Then, once fixed a decayingvacuum model, there are many DBI descriptions that lead to an equivalent dynamics for the universe. Among the great variety of possible choices, I pick here the simplest one, based on the assumption that both $\bar{\gamma}$ and $c_{s}$ are constant. Since $x$ is constant, because I want to assure that the underlying $\Lambda(t)$ and its corresponding DBI analog are thermodynamically equivalent, the three constant parameters $x, \bar{\gamma}$ and $c_{s}$ allow for a straightforward reconstruction of the functions $f(\phi)$ and the potential $V(\phi)$ appearing in the DBI Lagrangian (66). We tackle this particular example in the next section.

\section{B. A particular case: $c_{s}$ constant and $\Lambda=3 \beta H^{2}$}

Among the many proposals found in the literature to implement a vacuum-decaying model (see, in particular, the Table I in [18] for some examples), in this paper I specialize to the model characterized by the following phenomenological expression for $\Lambda(t)$ (see [14], 16], 18] and references therein):

$$
\Lambda(H) \equiv 3 \beta H^{2},
$$


where $\beta \in[0,1]$ is a dimensionless constant parameter. From (12) and (77) it is straightforward to show that such law yields $\bar{\gamma}=\gamma(1-\beta)$, which is a constant, as desired; next, I set $c_{s}$ in (67) to be a constant ${ }^{1}$. Then, with such constant parameters, the integral (76) can be easily carried out, and the result is

$$
a(\phi)=a_{*} e^{b\left(\phi-\phi_{*}\right)},
$$

where

$$
b \equiv \frac{1}{\sqrt{3} M_{P} \sqrt{\bar{\gamma} x c_{s}}} .
$$

From the definition of $\bar{\gamma}$, equation (12), we can rewrite the integral (76) in terms of $H$, which yields

$$
H(\phi)=H_{*} \exp \left[-\frac{3}{2} b \bar{\gamma}\left(\phi-\phi_{*}\right)\right] .
$$

Combining equations (78) and (80), we see that the Hubble parameter evolves as a power law of $a$,

$$
H(a)=H_{*}\left(\frac{a}{a_{*}}\right)^{-\frac{3}{2} \bar{\gamma}} .
$$

From expressions (67), (774) and (80) we find the corresponding expression for the function $f(\phi)$ :

$$
f(\phi)=\frac{1-c_{s}^{2}}{3 M_{P} \bar{\gamma} x c_{s} H_{*}} e^{3 b \bar{\gamma}\left(\phi-\phi_{*}\right)} .
$$

Lastly, from equations (42), (75) and (80) the potential can be easily evaluated, and yields

$$
V(\phi)=V_{*} e^{-3 b \bar{\gamma}\left(\phi-\phi_{*}\right)},
$$

where

$$
V_{*} \equiv 3 M_{P}^{2} H_{*}^{2}\left[1-\bar{\gamma}\left(\frac{x}{1+c_{s}}+\frac{1-x}{\gamma}\right)\right] .
$$

\footnotetext{
${ }^{1}$ Non-canonical scalar field models with constant speed of sound have already been studied in the literature. It is worth recalling that a DBI inflationary model with constant (and low) speed of sound has been discussed in 25]; also, another $k$-essence model with constant speed of sound different from unity has also been discussed in [5]
}

Therefore, a DBI model with exponential potential and constant speed of sound is dynamical- and thermodynamically equivalent to a vacuum-decaying model obeying the law $\Lambda(H)=3 \beta H^{2}$. Notice that the canonical result obtained in reference [21] is very similar to the one I derived here; the basic difference concerning the dynamical equations in both models is the presence of the speed of sound $c_{s}$, which is equal to one in the canonical version. Also, unlike the canonical results, in which $\bar{\gamma}$ and $x$ are the two free parameters to be constrained by observations, in this DBI model there is another free parameter, $c_{s}$, which can take either a small value (less than one) or even be superluminal ${ }^{2}$. Only a statistical analysis can constrain this space of free parameters, and this is the subject of a future work.

\section{CONCLUSIONS}

In this paper I generalize the approach devised in 21] to the case of noncanonical scalar fields. Following the first-order thermodynamical formalism developed to describe the decay mechanism of the canonical scalar field into particles [22], I derive the corresponding nonequilibrium relations for the noncanonical case, and obtain precisely the same equations, showing that noncanonical models are all thermodynamically equivalent among themselves and to an arbitrary $\Lambda(t)$ model by assuming that the specific entropy associated with each cosmic component is conserved (the "adiabatic" condition). Also, I find the general expression for the $x$ parameter, responsible for such thermodynamical equivalence.

As an illustration of the general procedure developed in this work to deal with noncanonical fields, I specialize to a DBI model with constant speed of sound. The formalism allows for a potential reconstruction, and I obtain a DBI model with exponential potential which is dynamical and thermodynamically equivalent to a decaying-vacuum model with the law $\Lambda(H)=3 \beta H^{2}$. In this particular case there are three parameters to be constrained by observations: the speed of sound associated with the noncanonical field, $c_{s}$, the $x$ and $\bar{\gamma}$ parameters. The statistical test to constrain such three parameters is the subject of a future work.

\footnotetext{
2 A solution with $c_{s}=1$ yields $x=0$ as seen from equation (46), which corresponds to the original $\Lambda(t)$ scenario.
} 


\section{Acknowledgments}

I thank Oswaldo Duarte Miranda and José Ademir Sales Lima for a critical reading of this paper.

[1] S. Perlmutter et al. [Supernova Cosmology Project Collaboration], Astrophys. J. 517, 565 (1999) [astro$\mathrm{ph} / 9812133]$.

A. G. Riess et al. [Supernova Search Team Collaboration], Astron. J. 116, 1009 (1998) [astro-ph/9805201].

[2] L. Perivolaropoulos, arXiv:0811.4684 [astro-ph].

[3] S. Weinberg, Rev. Mod. Phys. 61, 1 (1989).

[4] C. Wetterich, Nucl. Phys. B 302, 668 (1988).

B. Ratra, P. J. E. Peebles and, Phys. Rev. D 37, 3406 (1988).

R. R. Caldwell, R. Dave, P. J. Steinhardt and, Phys. Rev. Lett. 80, 1582 (1998) [astro-ph/9708069].

I. Zlatev, L. -M. Wang, P. J. Steinhardt and, Phys. Rev. Lett. 82 (1999) 896 [astro-ph/9807002].

[5] C. Armendariz-Picon, T. Damour and V. F. Mukhanov, Phys. Lett. B 458, 209 (1999) [arXiv:hep-th/9904075].

[6] C. Armendariz-Picon, V. F. Mukhanov, P. J. Steinhardt and , Phys. Rev. Lett. 85, 4438 (2000) [astro$\mathrm{ph} / 0004134]$.

[7] E. Silverstein and D. Tong, Phys. Rev. D 70, 103505 (2004) [arXiv:hep-th/0310221].

[8] M. Alishahiha, E. Silverstein and D. Tong, Phys. Rev. D 70, 123505 (2004) [hep-th/0404084].

[9] D. Bessada, W. H. Kinney and K. Tzirakis, JCAP 0909, 031 (2009) [arXiv:0907.1311 [gr-qc]].

[10] J. Martin, M. Yamaguchi and, Phys. Rev. D 77, 123508 (2008) [arXiv:0801.3375 [hep-th]].

[11] C. Ahn, C. Kim, E. V. Linder and , Phys. Lett. B 684, 181 (2010) [arXiv:0904.3328 [astro-ph.CO]].
[12] C. Ahn, C. Kim, E. V. Linder and, Phys. Rev. D 80, 123016 (2009) [arXiv:0909.2637 [astro-ph.CO]].

[13] O. Bertolami, Nuovo Cim. B 93, 36 (1986). M. Ozer and M. O. Taha, Nucl. Phys. B 287, 776 (1987).

[14] K. Freese, F. C. Adams, J. A. Frieman and E. Mottola, Nucl. Phys. B 287, 797 (1987).

[15] P. J. E. Peebles, B. Ratra and , Astrophys. J. 325, L17 (1988).

[16] J. C. Carvalho, J. A. S. Lima and I. Waga, Phys. Rev. D 46, 2404 (1992).

[17] J. A. S. Lima and J. C. Carvalho, Gen. Rel. Grav. 26, 909 (1994).

[18] J. M. Overduin and F. I. Cooperstock, Phys. Rev. D 58, 043506 (1998) [astro-ph/9805260].

[19] J. Sola, J. Phys. Conf. Ser. 283, 012033 (2011) [arXiv:1102.1815 [astro-ph.CO]].

[20] J. S. Alcaniz, J. A. S. Lima and, Phys. Rev. D 72, 063516 (2005) [astro-ph/0507372].

[21] J. M. F. Maia and J. A. S. Lima, Phys. Rev. D 65, 083513 (2002) [astro-ph/0112091].

[22] J. A. S. Lima, Phys. Rev. D 54, 2571 (1996) [grqc/9605055].

[23] R. Bean, D. J. H. Chung and G. Geshnizjani, Phys. Rev. D 78, 023517 (2008) [arXiv:0801.0742 [astro-ph]].

[24] I. R. Klebanov and M. J. Strassler, JHEP 0008, 052 (2000) [arXiv:hep-th/0007191].

[25] M. Spalinski, JCAP 0804, 002 (2008) [arXiv:0711.4326 [astro-ph]]. 